University of South Carolina

Scholar Commons

Publications

Artificial Intelligence Institute

4-19-2021

\title{
Cognitive Digital Twins for Smart Manufacturing
}

\author{
Muhammad Intizar Ali \\ Dublin City University \\ Pankesh Patel \\ University of South Carolina - Columbia \\ John G. Breslin \\ NUI Galway \\ Ramy Harik \\ University of South Carolina - Columbia \\ Amit Sheth \\ University of South Carolina - Columbia
}

Follow this and additional works at: https://scholarcommons.sc.edu/aii_fac_pub

Part of the Computer and Systems Architecture Commons, Hardware Systems Commons, Other

Computer Engineering Commons, Other Electrical and Computer Engineering Commons, and the Robotics Commons

\section{Publication Info}

Preprint version IEEE Intelligent Systems, 2021.

(c) IEEE Intelligent Systems 2021, IEEE

This Article is brought to you by the Artificial Intelligence Institute at Scholar Commons. It has been accepted for inclusion in Publications by an authorized administrator of Scholar Commons. For more information, please contact digres@mailbox.sc.edu. 


\section{Cognitive Digital Twins for Smart Manufacturing}

Muhammad Intizar Ali, Dublin City University, Ireland

Pankesh Patel, AI Institute, University of South Carolina, USA

John G. Breslin, Confirm SFI Research Centre for Smart Manufacturing, NUI Galway, Ireland

Ramy Harik, Mechanical Engineering, University of South Carolina, USA

Amit Sheth, AI Institute, University of South Carolina, USA

\section{Introduction}

Smart manufacturing or Industry 4.0, a trend initiated a decade ago, aims to revolutionize traditional manufacturing using technology-driven approaches. Modern digital technologies such as the Industrial Internet of Things (IloT), Big Data Analytics, Augmented/Virtual Reality, and Artificial Intelligence $(\mathrm{Al})$ are the key enablers of new smart manufacturing approaches.

The digital twin is an emerging concept whereby a digital replica can be built of any physical object. Digital twins are becoming mainstream; many organizations have started to rely on digital twins to monitor, analyze, and simulate physical assets and processes [1]. The current use of digital twins for smart manufacturing is largely limited to (i) status monitoring, (ii) simulation, and (iii) visualization. For status monitoring, digital replicas of physical assets (e.g., machines) are created, machines are continuously monitored using IloTs, and the latest status of a machine can be assessed by querying its digital twin. For simulation, digital twins of machines, processes, and products are created to mimic real settings. Simulation allows the design, development, and testing of new products and processes using their digital twins before applying them to actual physical assets, this is presented in [5]. For visualization, digital twins can include real-time dashboards and alert systems to monitor and debug an operational environment [2]. However, in contemporary cases, digital twins are simply considered to be an exact replica of the physical assets, without any value-added services built on top of them which could convert physical assets into autonomous intelligent agents. A major advantage of this enhanced design of digital twins is that they can offer much more than just an exact replica to support value-added services on top of digital twins, which are not possible on the physical assets.

\section{Cognitive Digital Twins}

Cognitive digital twins are an extension of existing digital twins with additional capabilities of communication, analytics, and intelligence in three layers: (i) access, (ii) analytics, and (iii) cognition.

The access layer is responsible for communication with the machine and gets access to data regarding the status of a physical asset to update the status of the digital twin. The analytics layer provides edge analytics capabilities at the device level. Similar to the edge analytics at the edge, this layer of the digital twin can perform additional analytical tasks on top of real-time collected data to help with the process of decision making by converting the raw sensory input 
into actionable knowledge [3]. The cognitive layer enables cognition by the digital twins. It is capable of performing complex decision making using edge analytics, domain expertise, and global knowledge bases. It is also responsible for communication among digital twins, allowing them to build their own networks and perform autonomous decision making. Cognitive digital twins will convert traditional digital twins into smart and intelligent agents that can access, analyze, understand, and react to their current status. In case of anomalies, rather than resorting to a simple alert system, the cognitive digital twin can interact with the operational environment and digital twins of products, running processes to further analyze and intelligently understand the anomalies. The cognitive digital twin can draw conclusions of situations locally and then also interact with other digital twins of physical assets operating in similar operational conditions to better understand shared local anomalies. Once identified, cognitive digital twins can interact socially with other peers and share knowledge and generate alerts in advance of any future potential unexpected situations. Insights from the analytics performed by cognitive digital twins will eventually help to build enterprise-level knowledge graph extraction, capture, and storage of domain knowledge.

Cognitive digital twins will disrupt existing technologies and applications used for digital twins by making them intelligent as well as social. The emerging concept of self-healing, self-configuring, and self-orchestrating systems is made possible using this approach. The team at the Confirm SFI Research Centre for Smart Manufacturing has implemented an initial prototype of cognitive digital twins using a benchmark dataset for production line performance monitoring [6] and intend to fully test the implemented prototype on the actual production lines of a smart factory in collaboration with an industry partner. An initial factory of the future to assess and implement this emerging concept is also being constructed at the University of South Carolina (Figure 1, see [7] for details). Having a social and interactive network of digital twins and a shared knowledge space will allow analytics and intelligence to go beyond the physical walls of a factory where digital twins can share their experience and lessons learned across the board. 


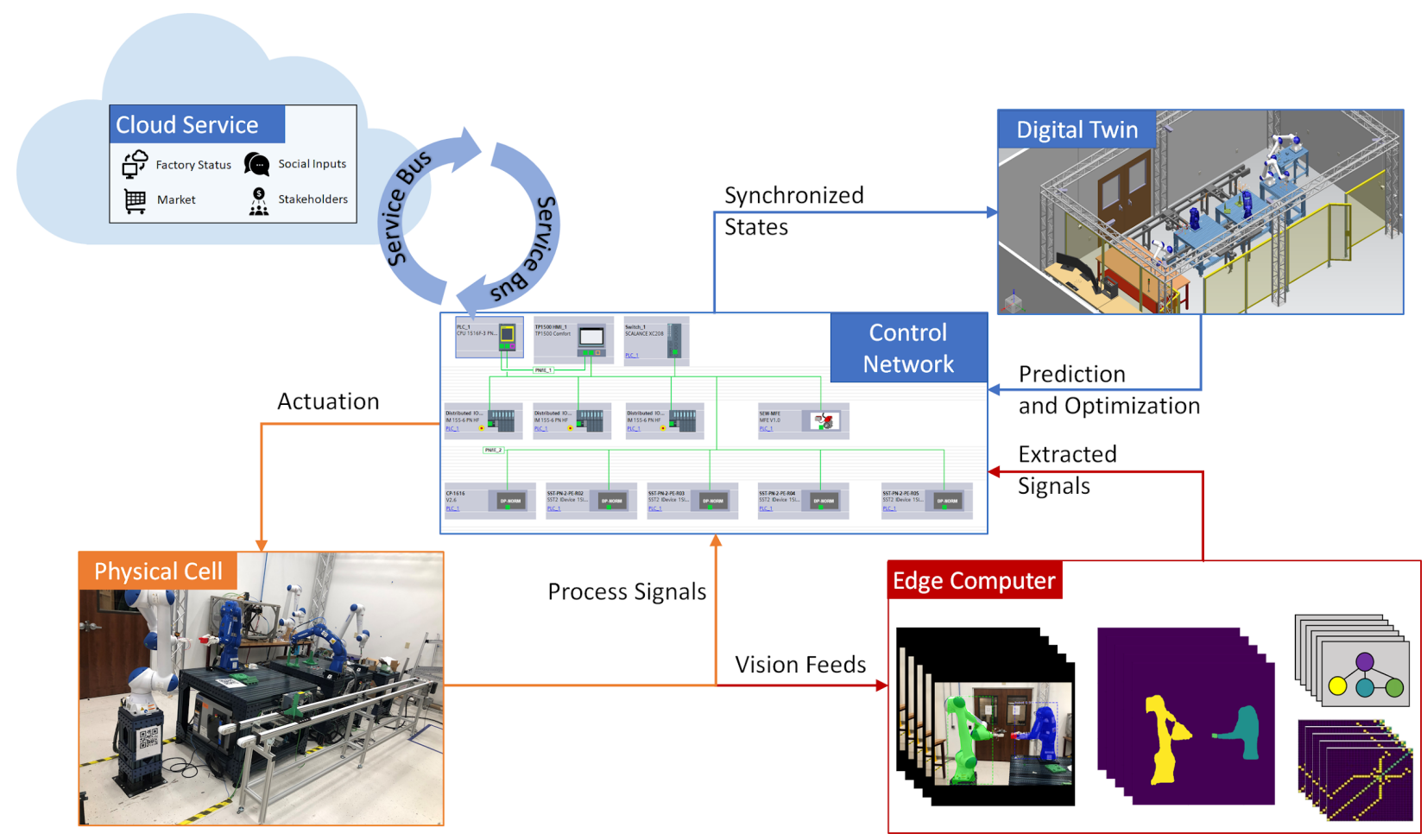

Figure 1: Proposed CPS-enabled control for future factories: control network administers physical cell and digital twin to synchronize process signals and intelligently actuate field devices by system smart layers. System smart layers consist of business intelligence from cloud services and semantic integration of visual signals from the edge ends.

\section{An Ecosystem of Cognitive Digital Twins}

We envision that once the cognitive digital twins are in place, they can build a network among themselves, having fully automated machine-to-machine interaction and decision making resulting in an ecosystem of cognitive digital twins. The knowledge gained by edge analytics, communication among digital twins and domain knowledge including user experiences will be captured as a unified knowledge graph. This knowledge graph will gradually evolve and will become a major source of information within the ecosystem of cognitive digital twins. Figure 2 presents a generic overview of cognitive digital twins ecosystems. We further elaborate our vision with an example use case of a manufacturing plant producing orthopedic implants, e.g. knee, hip and elbow joint replacements. On the shop floor, various machines are placed in an assembly line performing different operations, e.g. cutting, grinding, and polishing, etc. Each machine is equipped with different sensors to monitor its functional state, e.g. temperature, voltage, vibration, and rotation. A cognitive digital twin is created for all machines, products and processes. Collaboration and communication among the digital twins during decision making is conducted in four stages as follows. 


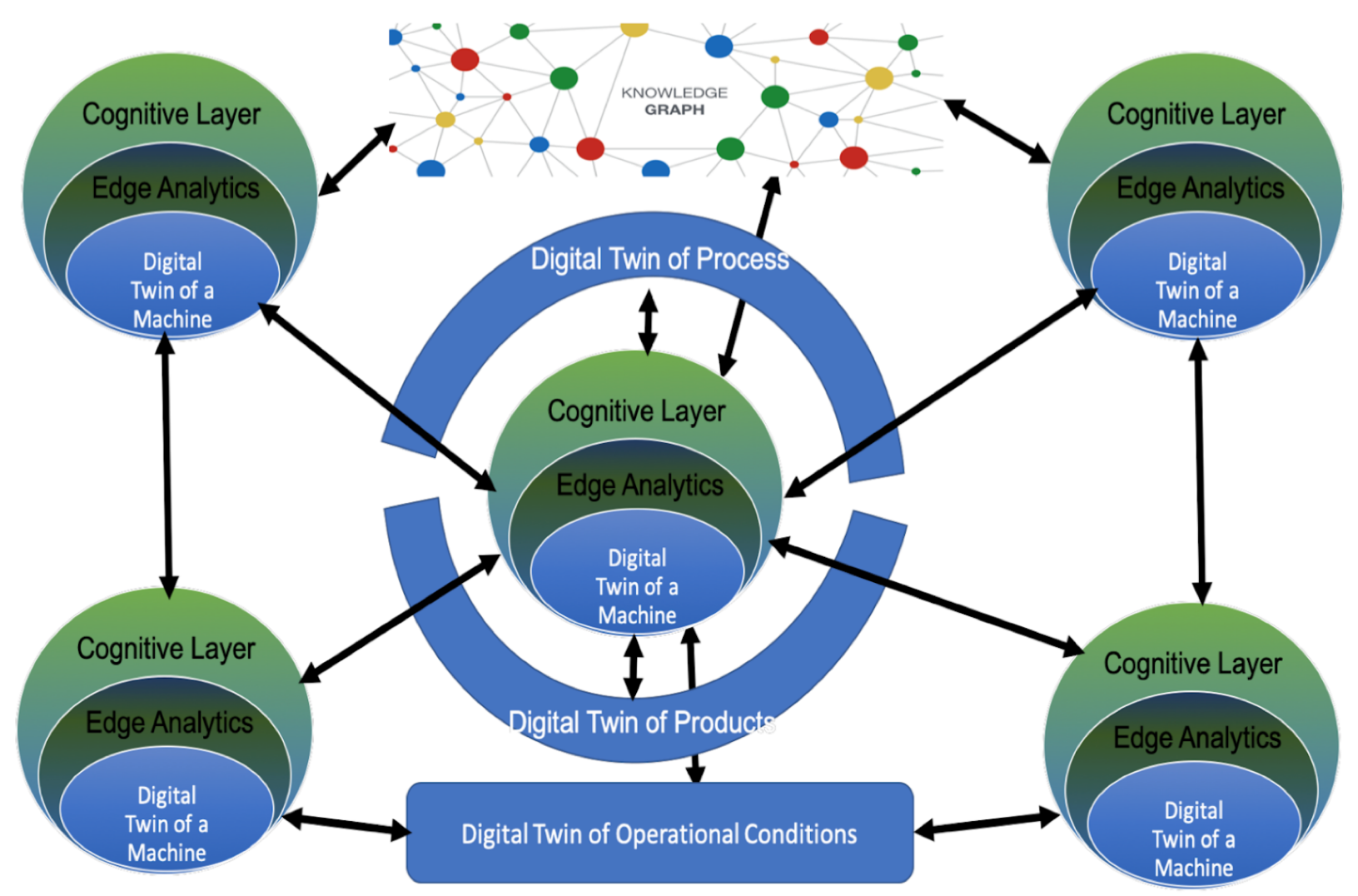

Figure 2: A Cognitive Ecosystem of Digital Twins

At the first stage, the cognitive digital twin of an industrial machine (e.g., a grinding machine) equipped with edge analytics is continuously monitoring values against predefined thresholds. An alert is created whenever a threshold is breached (e.g. the temperature of a motor inside the grinding machine goes beyond an acceptable threshold) [4]. At the second stage, the cognitive digital twin starts the sensemaking process by collecting contextual information including product characteristics (e.g. to check the rigidity of a metal alloy being used for a product), configurations of the processes being applied by the machine (e.g. pressure and speed of a grinding process), and operational conditions on the factory shop floor such as temperature, humidity, etc. The cognitive digital twins are capable of correlating all acquired information and initiating a sensemaking process to understand whether the current spike in temperature is due to a fault in the machine, characteristics of the product being manufactured, the manufacturing process being applied, or conditions on the shop floor. A factory level knowledge base is gradually created for all previous anomalies detected and their remedial actions. If a preexisting similar cause is identified, and its remedial action is available in the knowledge base, the cognitive digital twin will adjust its configuration, request a process adjustment, and/or adjust operational conditions accordingly. In the third stage, if a cognitive digital twin is unable to make sense of local information, it seeks further assistance from the social network of its peers and 
requests information from similar machines with similar operational conditions, e.g. a grinding machine of the same make and brand being used in a different plant. If an anomaly in temperature is only being observed locally, the digital twin of the machine adjusts itself to the configuration of machines running optimally without any issues. If the anomaly is observed across the board, a network-wide alert is broadcasted to request remedial actions. In the fourth stage, a record of captured events, interactions, the outcome of analytics, and the sensemaking process together with domain expertise is stored in a shared knowledge base in the shape of an enterprise-level knowledge graph. This knowledge graph will act as a central information portal for any future occurrences of similar events. We foresee that in the future, this knowledge graph will act as a central hub for all operational machines to post questions and get immediate answers. When necessary, a human expert may also be consulted.

\section{Research Challenges}

To realize the vision of cognitive digital twins, we envision a design and implementation of a distributed cross-domain autonomous system for smart manufacturing. The goal of this system is to enhance autonomous manufacturing by empowering manufacturing resources to think, learn and understand the dynamics of industrial environments by effectively integrating human cognition through $\mathrm{Al}$ and Semantic Web technologies into the design of autonomous manufacturing, respecting the Industry 4.0 system design principles. The approach can be cross-disciplinary, involving Al, semantic-empowered techniques, as well as semantic data integration in autonomous manufacturing scenarios. To achieve the above-mentioned vision, the following intertwined Research Questions (RQs) need to be addressed:

RQ1: How to create an autonomous distributed system conjoining the bottom-level manufacturing resources to enhance responsiveness and intelligence? This research question is further divided into the following research areas:

- A Collaborative Network of Intelligent Agents: This research investigates the design of an autonomous system that can discover and detect faults and disturbances autonomously as well as collaboratively. In addition to this, it can attempt to go beyond the existing knowledge of known problems to mitigate new problems and anomalies, thus capable of operating in unknown environments. Furthermore, they can build a collaborative network of intelligent agents locally to improve the responsiveness of the system.

- Automated Analytics for Resource-constrained Manufacturing Resources[8]: This research requires the investigation of the suitability of existing interoperability standards (e.g., Web of Things, RAMI 4.0, Semantic Web) and the suitability of existing architecture patterns (e.g., fog, Intelligent edge [3], and smart agent) for resource-constrained manufacturing resources as it demands quick response and automatic analytics with enhanced intelligent capabilities. 
- Autonomous Models on top of Knowledge Graph: This research requires investigation of incorporating several autonomous models on top of semantic-empowered technologies as we do not want to limit our vision of cognitive digital twins only for a specific autonomous model. For instance, an integration of self-comparison models, where a single machine can be compared with a fleet of similar machines. This capability can be extended further by leveraging historical information to predict its suitability for autonomous resource allocation.

RQ2: How to enable an autonomous cross-domain reasoning over distributed Industry 4.0 applications?

Industry 4.0 applications are currently designed while keeping a single application domain in view. Most of these applications target a domain-specific problem. Cross-domain collaborations allow to deduce additional events from a silo and can be turned into useful actuation, e.g., before allocating manufacturing resources, a system considers external electricity rates and supply chain data (e.g., weather and traffic conditions) in order to achieve the goal of reducing the factory's energy consumption and carbon footprint.

To address this research question, we need to investigate an autonomous cross-domain system, which can leverage semantic reasoning to derive new knowledge and Al techniques to monitor and process events from totally independent applications. It can integrate the techniques of knowledge discovery and inference that is not possible from data generated by a single application. Moreover, it can use algorithms for autonomous decision-making with uncertain, dynamic and incomplete information. Having a framework among industrial machines and shared collaborative intelligence identified in RQ1 can prepare the necessary ground to achieve RQ2, synthesizing analytics and intelligence of factories with other external knowledge and services for decision-making.

\section{ACKNOWLEDGMENTS}

This publication has emanated from research supported by grants from the European Union's Horizon 2020 research and innovation programme under grant agreement number 847577 (SMART 4.0 Marie Sklodowska-Curie actions COFUND) and from Science Foundation Ireland (SFI) under grant number SFI/16/RC/3918 (Confirm) co-funded by the European Regional Development Fund, and support from SCRA (South Carolina Research Authority).

\section{References}

[1] Shiva Sander Tavallaey and Christopher Ganz, "Automation to Autonomy", 24th IEEE International Conference on Emerging Technologies and Factory Automation (ETFA), 2019.

[2] Muhammad Intizar Ali, Pankesh Patel, Soumiya Kanti Datta, and Amelie Gyrard, "Multi-Layer Cross Domain Reasoning over Distributed Autonomous IoT Applications," the International Workshop on Very Large Internet of Things (VLIoT 2017) in conjunction with the VLDB, 2017.

[3] Pankesh Patel, Muhammad Intizar Ali, and Amit Sheth, "From Raw Data to Smart 
Manufacturing: Al and Semantic Web of Things for Industry 4.0", IEEE Intelligent Systems, 2018.

[4] Vignesh Kamath, Jeff Morgan, and Muhammad Intizar Ali, "Industrial loT and Digital Twins for a Smart Factory: An open source toolkit for application design and benchmarking," IEEE Global Internet of Things Summit (GloTS), 2020.

[5] Kaishu Xia, Christopher Sacco, Max Kirkpatrick, Clint Saidy, Lam Nguyen, Anil Kircaliali and Ramy Harik, "A digital twin to train deep reinforcement learning agent for smart manufacturing plants: Environment, interfaces and intelligence", Journal of Manufacturing Systems, 2020.

[6] Pankesh Patel and Muhammad Intizar Ali, "Developing Real-time Smart Industrial Analytics for Industry 4.0 Applications," Smart Service Management - Design Guidelines and Best Practices, Springer, 2020.

[7] Kaishu Xia, Clint Saidy, Max Kirkpatrick, Noble Anumbe, Amit Sheth and Ramy Harik, "Semantic integration of machine vision systems to aid manufacturing event understanding", Journal of Manufacturing Systems, Submitted, 2020.

[8] Bararath Sudarsan, Pankesh Patel, Muhammad Intizar Ali, John Breslin, and Rajiv Ranjan, "Towards Executing Neural Networks-based Video Analytics Models on Resource-Constrained IoT Devices", IEEE Internet Computing, Submitted, 2020. 